\title{
MULTILAYERED POLYMER MICROFLUIDIC CHIP WITH NANOFLUIDIC INTERCONNECTS FOR MOLECULAR MANIPULATION
}

\author{
Bruce R. Flachsbart ${ }^{1,2}$, Kachuen Wong ${ }^{1}$, Jamie M. Iannacone ${ }^{2,3}$, Edward N. Abante ${ }^{1}$, Robert L. Vlach ${ }^{1}$, Peter A. \\ Rauchfuss ${ }^{1}$, Paul W. Bohn ${ }^{2,3}$, Jonathan V. Sweedler ${ }^{2,3}$, and Mark A. Shannon ${ }^{1,2}$ \\ ${ }^{1}$ Department of Mechanical \& Industrial Engineering, University of Illinois at Urbana-Champaign \\ ${ }^{2}$ Beckman Institute for Advanced Science and Technology, University of Illinois at Urbana-Champaign \\ ${ }^{3}$ Department of Chemistry, University of Illinois at Urbana-Champaign \\ Urbana, Illinois USA
}

\begin{abstract}
The design, fabrication, and preliminary testing is presented for a polymer multilayered hybrid micro-nanofluidic chip that consists of poly(methylmethacrylate) (PMMA) layers containing microfluidic channels separated in the vertical direction by polycarbonate (PC) nanocapillary array membranes (NCAMs). This design architecture enables nanofluidic interconnections to be placed in the vertical direction between microfluidic channels. Such an architecture combines microfluidic manipulations (separation, injection, collection, etc.) with nanofluidic molecular capabilities (molecular sizing and affinity reactions, channel isolation, enhanced mixing, etc.) on a single chip. Recent polymeric microfabrication advances have made this scalable construct possible: 1) processing thin polymer layers on releasable and compliant carriers, and 2) the high resolution contact-printing of a strong thermal adhesive. Bond strength was demonstrated by pressurizing channels with 90 psi nitrogen without failure. Devices were characterized in terms of measuring resistivity and electroosmotic flow (EOF) along the channels at different $\mathrm{pH}$ values. The functionality of the chip is demonstrated by filling a cross channel with $1 \mu \mathrm{M}$ green-fluorescent protein (GFP) and electrokinetically transporting analyte plugs through the NCAM and down the separation channel while performing laser induced fluorescence (LIF) analysis. The development of this new type of hybrid micro-nanofluidic device potentially will allow unprecedented molecular manipulations for chemical and biological sensing applications.
\end{abstract}

\section{INTRODUCTION}

Three dimensional (multilayered) microfluidic devices have been pursued in order to increase the functionality and complexity of the sequential biochemical manipulations possible within a device [1-4]. A proposed approach to further increase the functionality of this class of device is integrating microfluidics with nanofluidics in a hybrid approach that can exploit the physical dimensions of the nanoscale through the incorporation of nanocapillary array membranes (NCAMs) within the device [5]. This approach has been demonstrated in polydimethylsiloxane (PDMS) based microfluidic devices and has been shown to facilitate a variety of sample manipulations, including: nanofluidic gated injection of an analyte and the electrophoretic separation of that analyte [6], the mixing and reaction an analyte and a reagent [7], the collection of a specific electrophoretically separated band [8], and the separation of an analyte based on mass (or molecular size) [9]. Here, we demonstrate this hybrid approach within a PMMA microfluidic device in a scalable construct incorporating multiple NCAMs.

Multilayered polymer microfluidic chips with nanofluidic interconnects, such as those shown in Fig. 1, were fabricated by sequentially bonding microfluidic layers separated in the vertical direction by NCAM layers. An expanded view of a device is given in Fig. 2 showing the individual layers, their function, and the bond order. In Fig. 2, layer 2 is first bonded to layer 1, and then layer 3 is added, and so on, in a top-down fabrication approach. The routing of the microfluidic channels between the reservoirs is depicted in Fig. 3 along with a cross-sectional view of a channel intersection region showing the nanofluidic interconnects between the crossing microfluidic channels. Both ends of the chips are symmetric, containing two sets of crosschannel nanofluidic interconnects for the purpose of injecting and collecting analyte bands to and from the $28 \mathrm{~mm}$ separation channel in the middle. All optically transparent materials, including the adhesive, were used, thus allowing both visible and near UV inspection and interrogation of all the channel layers.

The fabrication process involved two recent advances in polymeric microfabrication: processing thin $(<40 \mu \mathrm{m})$, labile polymer layers on a compliant carrier that is released after bonding, and contact-printing a high resolution $(<100 \mu \mathrm{m})$ custom adhesive. Thin microfluidic layers are fragile and by supporting them on carrier substrates, extensive further processing becomes possible (metallization, etch, deposition, patterning, etc.). The compliance of this construct (layer plus carrier) is an important issue when bonding multi-layered structures. Bonds must accommodate both local non-uniformities (defects, submicron debris, etc.), and global non-uniformities (surface warpage, curvature, etc.), with the later tending to be additive with each layer. PMMA and PC have moduli 2-3 orders of magnitude greater than PDMS, and to achieve good bond quality a combination of adhesive layer compliance, for local nonuniformities, and layer carrier compliance, for global nonuniformities, was used. The second fabrication advance incorporated the contact printing of an adhesive layer using a flat

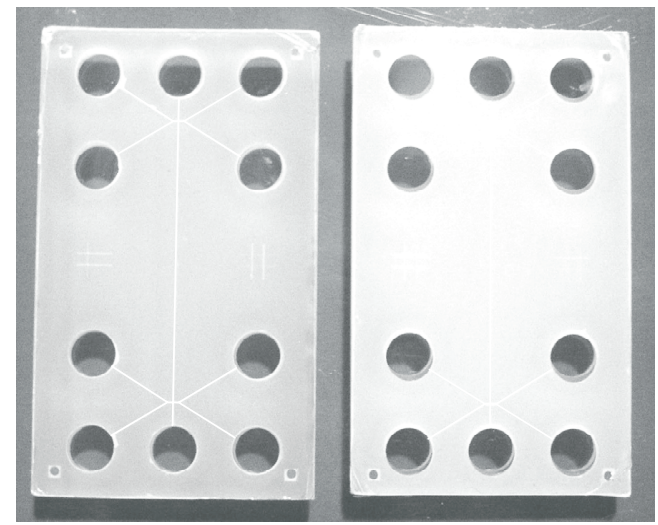

Figure 1 A multilayer device (eight layers in total) containing three microfluidic channel layers separated vertically by two nanocapillary array membranes (NCAMs). The NCAMs within each device are 10 and $100 \mathrm{~nm}$ for the left sample, and 10 and 220 $\mathrm{nm}$ for the right sample. Chips are $24 \times 40 \mathrm{~mm}$ ( 1.6 mm thick). 


\section{Layer Description}

1 Polycarbonate reservoir layer with ten $4 \mathrm{~mm}$ diameter reservoirs

2 PMMA via layer which couple reservoirs to the microfluidic channels

3 PMMA channel layer for sample introduction or collection

$4 \quad$ NCAM

5 PMMA separation channel layer

6 NCAM
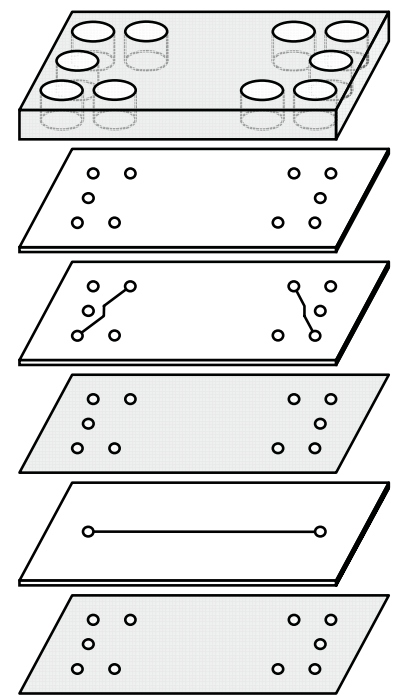

7 PMMA channel layer for sample introduction or collection

8 PMMA bottom layer

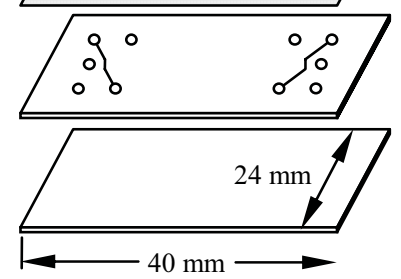

Figure 2 Diagram depicting the individual layers of the 8-layer devices shown in Fig. 1. PMMA and NCAMs range in thickness from 5 to $40 \mu \mathrm{m}$ and 6 to $10 \mu \mathrm{m}$ respectively.

disk-shaped PDMS stamp. While elastomeric stamps have been used to contact print monolayer inks [10], thin metal films [11], and liquid polymers [12], the use of contact printing in microelectromechanical system (MEMS) fabrication to pattern layers as thick as $1 \mu \mathrm{m}$, as in the adhesive layer printing of benzocyclobutene for wafer level bonding [13], is relatively recent. PDMS is commonly used for contact printing primarily due to its ability to conform to a surface without trapping bubbles at the interface [14].

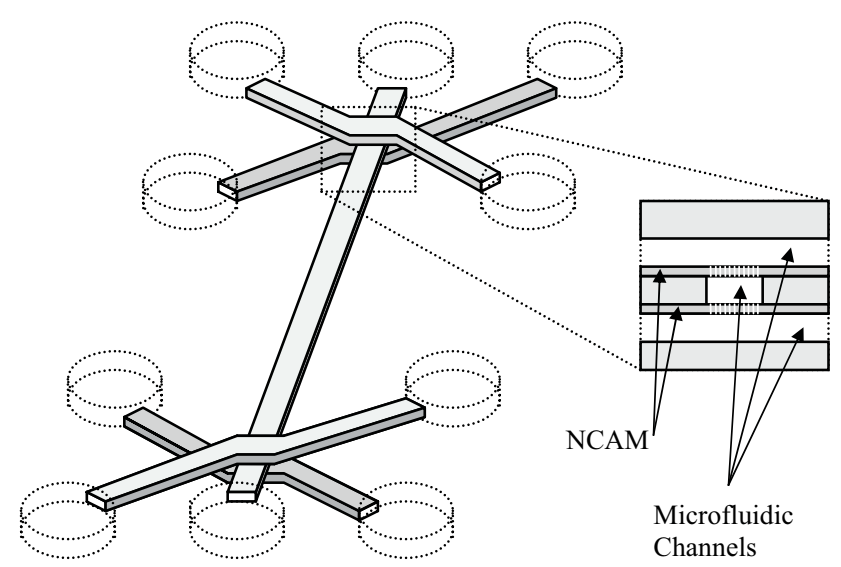

Figure 3 Relief schematic of an eight layer device showing the routing scheme for the microfluidic channels relative to each other and the reservoirs. On the right is a cross-section of the intersection region where nanofluidic interconnects is formed between the microfluidic layers via NCAMs.

\section{DEVICE FABRICATION}

The assembly of the layers into the device in Fig. 1 consisted of the sequential operations of contact printing adhesive layers, bonding, and releasing the bonded PMMA layers from their temporary carriers. The overall fabrication scheme of the multilayer device is shown in Fig. 2 and consisted of: (a) a semirigid PC reservoir layer on which to build the device; (b) individually processing each distinct labile polymer layer on a separate carrier, including if necessary spinning and curing the polymer layer, patterning, etching, and applying the adhesive; (c) transferring, aligning, and bonding the labile polymer layer to the device; (d) releasing the carrier; and (e) repeating with subsequent layers to form a multilayer chip. First, adhesive was contact printed onto the top surface of PMMA layer \#2 in Fig. 2, which was then bonded to the PC top piece (layer \#1 in Fig. 2) at $130^{\circ} \mathrm{C}$ and 5.2 $\mathrm{MPa}$ of applied pressure under vacuum for 10 minutes. PMMA layer \#2 was processed while affixed to a temporary coverglass carrier (No. 2 thickness), which, after bonding, was released by submersion in a hot water bath at approximately $50^{\circ} \mathrm{C}$ for $5 \mathrm{~min}$. Layer compliance was achieved using an elastomeric polymer to press the coverglass to the sample stack with $5.2 \mathrm{MPa}$ of force at $130^{\circ} \mathrm{C}$. The next PMMA layer $\# 3$ was bonded to the device stack in the same way that layer \#2 was bonded (i.e. the top surface of PMMA layer \#3 was coated with an adhesive, whereby it was bonded to the device stack, and its temporary carrier released using a hot water bath). Bonding NCAM layers required a slightly different approach since adhesive could not be applied to the NCAM layer without plugging the nanoscale pores. Thus the adhesive was applied to each of the layers facing the NCAM layer. Accordingly, the bottom surface of PMMA layer \#3 and the top surface of the PMMA layer \#5 were coated with adhesive. An NCAM layer \#4 was placed between them, aligned and bonded together. After the bonding process, the coverglass carrier for PMMA layer \#5 was released. The process was repeated for the second NCAM layer \#6 and the PMMA layer \#7. The final, unpatterned PMMA layer \#8 was bonded to the device after coating the bottom of PMMA layer \#7. The final step was a $12 \mathrm{hr}$. vacuum-oven cure at $130^{\circ} \mathrm{C}$ at a temperature and time sufficient to fully crosslink all the epoxy adhesive layers without allowing remaining solvents or curing byproducts to coalesce.

The PMMA layers were formed by spincoating PMMA dissolved in propylene glycol monomethyl ether acetate (PGMEA) and anisole (all from Sigma Aldrich) onto a coverglass (Fisher Scientific, $35 \times 50 \mathrm{~mm}$, \#2 thickness) that acted as the carrier plate for the PMMA layer. The PMMA on the coverglass was then cured to $180^{\circ} \mathrm{C}$ in an oven for 6 to 24 hours depending on layer thickness. After curing, the PMMA layer was sputter coated with a layer of aluminum $(\sim 100 \mathrm{~nm})$, and patterned using standard photolithographic procedures. The development of the positive photoresist etched the aluminum layer, thereby transfering the mask pattern to the aluminum layer. The PMMA channels and vias were then formed by reactive ion etching (RIE) using an oxygen and argon plasma (Axic RIE - 600 Watts). A straight side-wall profile created during this etch step was important for high resolution adhesive transfer. The final step in the preparation of the PMMA layers was the removal of the aluminum layer with photoresist developer, which also removed any remaining photoresist residue.

NCAM layers are commercially available from GE Osmonics Labstore and can be obtained with nominal pore diameters ranging from $10 \mathrm{~nm}$ to $400 \mathrm{~nm}$. NCAMs are temporarily mounted onto PDMS disks using methanol, so that they lie flat when being 
aligned and transferred to the device. Figure 4 shows a $47 \mathrm{~mm}$ diameter NCAM layer after bonding showing complete coverage of the underlying $24 \times 40 \mathrm{~mm}$ device. The NCAM layer would then be trimmed and openings the size of the reservoirs made using RIE and a silicon shadow mask.

Figure 4 Device after first NCAM layer bonded, showing the complete overlap of the $24 \times 40 \mathrm{~mm}$ device by the $47 \mathrm{~mm}$ diameter NCAM. The excess NCAM would then be trimmed before bonding the next layer to the device.

\section{ADHESIVE CONTACT PRINTING}

The transfer process consisted of spincoating a PDMS substrate (disk) with adhesive, which was then transferred by pressing the adhesive onto the desired layer to be bonded. The epoxy adhesive was a mixture of Dow Corning solid epoxy novalac-modified resin with curing agent in a 2.5:1 mass ratio, and various solvents (2-methoxyethanol 15 to $50 \%$ by mass range, anisole 15 to $50 \%$ by mass range, and PGMEA 0 to $10 \%$ by mass range, the exact amounts depend upon the adhesive layer thickness desired). Most often, the solvents were selected to modify the viscosity of the adhesive in order to achieve a thickness of $\sim 1 \mu \mathrm{m}$ via spincoating in order to achieve sharp transfer interfaces.

Optical microscope inspection after contact printing was used to monitor the degree to which the pattern was resolved during the contact printing procedure. Figure 5 depicts a PDMS substrate after contact printing to layer \#7, and both the PDMS substrate and the device are utilized for quality control. Ultimate printing resolution was determined by the smallest dimension that could be printed consistently without "bridging" and/or seeping of the adhesive into the channel. Figure 6 shows some examples of common contact printing errors. If a printed layer had errors, the PMMA surface could be reprinted with adhesive after removing the previous layer with methanol. Features smaller than $100 \mu \mathrm{m}$ can be resolved using an adhesive layer approximately $1 \mu \mathrm{m}$ thick.

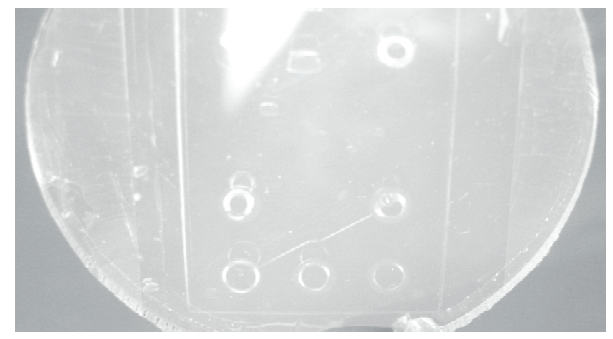

Figure 5 A photograph of a PDMS substrate after contact printing an adhesive layer onto a PMMA microfluidic channel layer. The adhesive pattern that is left on the PDMS can be monitor to verify the quality of the transfer process.
Thinner adhesive layers generally achieve better transfer resolution, but also tend to be harder to release from the PDMS substrate and do not accommodate local non-uniformities as well.

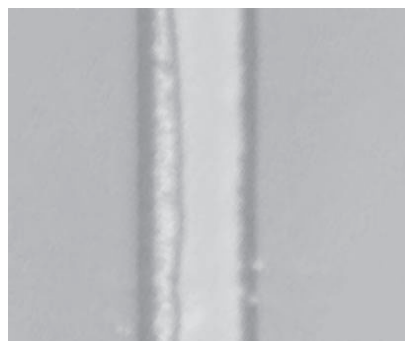

(a)

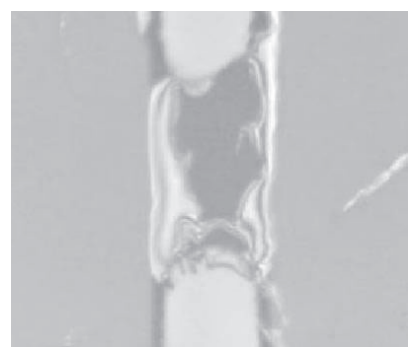

(b)
Figure 6 Optical images of microfluidic channels showing contact printing errors where (a) the adhesive has seeped into the channel, and (b) the adhesive has "bridged" the microchannel.

Another factor that affected contact printing resolution was the temperature of the adhesive carrier. PDMS has a greater affinity to the adhesive layer when it is cold, and the affinity decreases with increasing temperature. Heating the PDMS carrier and adhesive to $50^{\circ} \mathrm{C}$ for $3 \mathrm{~min}$ improved the transfer of adhesive to the areas in contact with the PMMA surface. The chip and adhesive carrier could then be cooled to improve the adhesion of the adhesive that was not in contact with the surface. This heating and cooling of the adhesive carrier substantially improved the yield of the process in addition to significantly improving the contact printing resolution.

\section{DEVICE TESTING}

Devices were tested physically, electrically, and functionally. The physical strength of the bonding process was tested by threading reservoirs to accommodate pressure hose fittings in a modified device as shown in Figure 7. Channels were then filled with a fluorescent solution and both ends pressurized with nitrogen while inspecting the channels for delamination. Reservoir bottom rupturing was the primary failure mechanism, generally occurring above $6 \mathrm{~atm}$ (90 psi). Electrical characterization consisted of 1) measuring microfluidic channel resistivity and 2) determining the electroosmotic flow (EOF) coefficients along the channels at different $\mathrm{pH}$ values. Linear $\mathrm{I}-\mathrm{V}$ plots $\left(\mathrm{R}^{2}>0.995\right)$ using $10 \mathrm{mM}$ phosphate buffer (PB) $(\mathrm{pH}$ 7.4) were obtained yielding a mean of $26.7 \pm 0.4 \mathrm{M} \Omega / \mathrm{cm}$ along the longer microfluidic channel $(\Delta l=2.80$ $\mathrm{cm})$ and $37.6 \pm 0.4 \mathrm{M} \Omega / \mathrm{cm}$ along the shorter cross-channels $(\Delta l=$ $1.23 \mathrm{~cm})$. Measurements were stable and reproducible over a

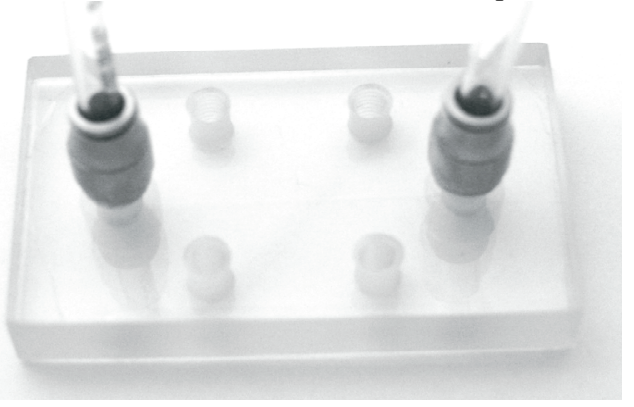

Figure 7 A chip modified to accommodate threaded highpressure hose connections used for pressure testing layer bond strength. The device was vacuum filled with fluorescent solution, then pressurized with nitrogen, while continuously inspecting the channel for delamination. Reservoir bottom rupturing was the primary failure mechanism above 90 psi. 
week. No measurable leakage current was observed through the chip itself, indicating no discernable fluid leaks between levels. Electroosmotic flow (EOF) mobilities versus solution $\mathrm{pH}$, given in Table 1, were measured using the current monitoring method using $5 \mathrm{mM}$ and $10 \mathrm{mM}$ PB solutions at $100 \mathrm{~V}$. These EOF values are within a factor of two of published EOF values for PMMA microfluidic channels, which are noted to vary with processing method [15]. Transport through the nanofluidic interconnects (across the NCAM membrane) was demonstrated by filling a cross channel with $1 \mu \mathrm{M}$ of green-fluorescent protein (GFP) and electrokinetically transporting analyte plugs (with $600 \mathrm{~V}$ pulses 2 seconds in duration) into the middle separation channel. A $488 \mathrm{~nm}$ $\mathrm{Ar}+$ laser was focused on the receiving channel away from the intersection and a detector was used to produce the laser induced fluorescence (LIF) graph shown in Figure 8. Plug injections were confined and reproducible.

Table 1 Electroosmotic coefficients $\left\{\mathrm{cm}^{2} / V \cdot s\right\}$ for phosphate buffer solution versus $\mathrm{pH}$ measured using the current monitoring method.

\begin{tabular}{|c|c|c|}
\hline & Cross Channel Ave. & Separation Channel \\
\hline $\mathrm{pH} 8.8$ & $2.7 \pm 0.6 \times 10^{-4}$ & $2.2 \pm 0.3 \times 10^{-4}$ \\
\hline $\mathrm{pH} 7.3$ & $2.8 \pm 0.5 \times 10^{-4}$ & $3.5 \pm 0.7 \times 10^{-4}$ \\
\hline $\mathrm{pH} 4.4$ & $2.5 \pm 0.9 \times 10^{-4}$ & $2.2 \pm 0.3 \times 10^{-4}$ \\
\hline
\end{tabular}

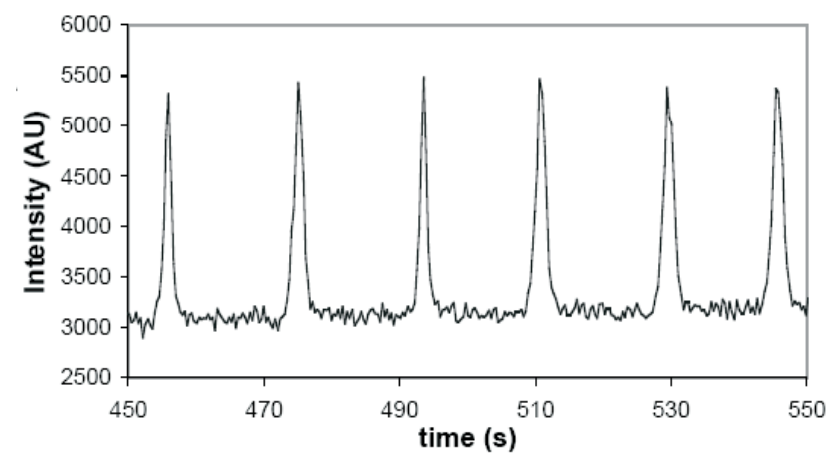

Figure 8 Laser-induced fluorescence (LIF) detection of $1 \mu M$ $G F P$ in $10 \mathrm{mMPB}$ injected for $2 \mathrm{~s}$ at $600 \mathrm{~V}$ from one microfluidic channel, across a NCAM, and into a second microfluidic channel containing the PB solution only.

\section{CONCLUSIONS}

The fabrication and preliminary testing of a hybrid micronanofluidic device using multiple NCAMs and PMMA microfluidic layers was detailed. This construct produced a high quality device that has the potential to be scaled beyond the eight layer devices presented and tested. The interconnection of five separate microfluidic channels with four nanofluidic interconnects seeks to increase the functionality and sophistication of the sequential biochemical manipulations possible within a device.

\section{ACKNOWLEDGEMENTS}

This work was partially supported by National Science Foundation's The WaterCAMPWS, a Science and Technology Center of Advanced Materials for the Purification of Water with Systems under the agreement number CTS-0120978, the Center for Nano-Chemical-Electrical-Mechanical Manufacturing Systems under DMI-032-28162, and by Strategic Environmental Research and Development Program under Army W9132T-05-2-0028.

\section{REFERENCES}

[1] T. Thorsen, S. J. Maerki, and S. R. Quake, "Microfluidic large-scale integration," Science, 298, p. 580-4, 2002.

[2] J. R. Anderson, D. T. Chiu, R. J. Jackman, O. Cherniavskaya, J. C. McDonald, H. Wu, S. H. Whitesides, and G. M. Whitesides, "Fabrication of topologically complex threedimensional microfluidic systems in PDMS by rapid prototyping," Analytical Chemistry, 72, p. 3158-3164, 2000.

[3] G. Mensing, T. Pearce, and D. J. Beebe, "An ultrarapid method of creating 3D channels and microstructures," JALA Journal of the Association for Laboratory Automation, 10, p. 24-28, 2005.

[4] B. H. Weigl, R. Bardell, T. Schulte, F. Battrell, and J. Hayenga, "Design and rapid prototyping of thin-film laminate-based microfluidic devices," Biomedical Microdevices, 3, p. 267-74, 2001.

[5] T.-C. Kuo, D. M. Cannon Jr, M. A. Shannon, P. W. Bohn, and J. V. Sweedler, "Hybrid three-dimensional nanofluidic/microfluidic devices using molecular gates," Sensors and Actuators, A: Physical, 102, p. 223-233, 2003.

[6] D. M. Cannon Jr, T.-C. Kuo, P. W. Bohn, and J. V. Sweedler, "Nanocapillary array interconnects for gated analyte injections and electrophoretic separations in multilayer microfluidic architectures," Analytical Chemistry, 75, p. 2224-2230, 2003.

[7] T.-C. Kuo, H.-K. Kim, D. M. Cannon Jr, M. A. Shannon, J. V. Sweedler, and P. W. Bohn, "Nanocapillary arrays effect mixing and reaction in multilayer fluidic structures," Angewandte Chemie - International Edition, 43, p. 18621865, 2004.

[8] J. J. Tulock, M. A. Shannon, P. W. Bohn, and J. V. Sweedler, "Microfluidic separation and gateable fraction collection for mass-limited samples," Analytical Chemistry, 76, p. 64196425, 2004.

[9] T.-C. Kuo, D. M. Cannon Jr, Y. Chen, J. J. Tulock, M. A. Shannon, J. V. Sweedler, and P. W. Bohn, "Gateable nanofluidic interconnects for multilayered microfluidic separation systems," Analytical Chemistry, 75, p. 1861-1867, 2003.

[10] L. H. Dubois and R. G. Nuzzo, "Synthesis, structure, and properties of model organic surfaces," Annual Review of Physical Chemistry, 43, p. 437-463, 1992.

[11] L. Yueh-Lin, R. L. Willett, K. W. Baldwin, and J. A. Rogers, "Additive, nanoscale patterning of metal films with a stamp and a surface chemistry mediated transfer process: applications in plastic electronics," Applied Physics Letters, 81, p. 562-4, 2002.

[12] M. Wang, H. G. Braun, T. Kratzmuller, and E. Meyer, "Patterning polymers by micro-fluid-contact printing," Advanced Materials, 13, p. 1312-1317, 2001.

[13] J. Oberhammer and G. Stemme, "BCB contact printing for patterned adhesive full-wafer bonded 0-level packages," Journal of Microelectromechanical Systems, 14, p. 419-425, 2005.

[14] Y. Xia and G. M. Whitesides, "Soft Lithography," Annual Review of Physical Chemistry, 28, p. 153-184, 1998.

[15] Z. Chen, Y. Gao, R. Su, C. Li, and J. Lin, "Fabrication and characterization of poly(methyl methacrylate) microchannels by in situ polymerization with a novel metal template," Electrophoresis, 24, p. 3246-3252, 2003. 